\title{
Diagnosis of steatohepatitis and fibrosis in biopsy-proven nonalcoholic fatty liver diseases: including two-dimension real-time shear wave elastography and noninvasive fibrotic biomarker scores
}

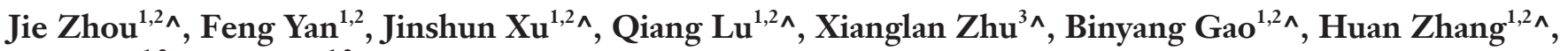 \\ Rui Yang ${ }^{1,2} \wedge$, Yan Luo ${ }^{1,2} \wedge$ \\ ${ }^{1}$ Ultrasound Department of West China Hospital of Sichuan University, Chengdu, China; ${ }^{2}$ Laboratory of Ultrasound Imaging of West China \\ Hospital of Sichuan University, Chengdu, China; ${ }^{3}$ Pathology Department of West China Hospital of Sichuan University, Chengdu, China
}

Contributions: (I) Conception and design: Y Luo; (II) Administrative support: Y Luo; (III) Provision of study materials or patients: Y Luo; (IV) Collection and assembly of data: J Zhou; (V) Data analysis and interpretation: J Zhou, Q Lu; (VI) Manuscript writing: All authors; (VII) Final approval of manuscript: All authors.

Correspondence to: Yan Luo, MD, PhD. Ultrasound Department of West China Hospital of Sichuan University, Chengdu, China.

Email: luoyanddoc@163.com.

\begin{abstract}
Background: The aim of this retrospective study was to evaluate the accuracy of two-dimension realtime shear wave elastography (2D-SWE) for the diagnosis of steatohepatitis and fibrosis in a cohort patients confirmed nonalcoholic fatty liver diseases (NAFLD) by liver biopsy, and compare with four noninvasive fibrotic biomarker scores (NFS, FIB-4, BARD and APRI).

Methods: 116 NAFLD patients and 23 normal control group were enrolled. The diagnostic performance of 2D-SWE and four noninvasive fibrotic biomarker scores was evaluated based on histopathological inflammation grades and fibrosis stages (F) according to Kleiner/Brunt et al.'s criteria classification. 5 -fold cross validation and receiver operating characteristics curve (ROC) analyses were used to obtain an assessment of 2D-SWE and four noninvasive fibrotic biomarker scores; then cross validated area under the curves (AUCs) were compared using the test of Delong. Meanwhile, influence of steatosis on liver stiffness measurement (LSM) of 2D-SWE was also studied.

Results: Liver stiffness measured by 2D-SWE proved to be an excellent diagnostic indicator for detecting steatohepatitis (AUROC $=0.88)$, and fibrosis: $\geq F 2$ stage $(A U R O C=0.86), \geq F 3$ stage $(A U R O C=0.89)$ and =F4 stage (AUROC $=0.90$ ) with the cutoff values were 7.3, 10.0, 11.6 and $12.6 \mathrm{kPa}$, respectively. Compared with fibrotic scores, 2D-SWE had the highest AUROC for predicting $\geq \mathrm{F} 2, \geq \mathrm{F} 3,=\mathrm{F} 4$ by Delong test $($ all $\mathrm{P}<0.05)$. No statistic differences of LSM were found among different steatosis levels ( $\mathrm{P}=0.97)$.

Conclusions: The stiffness measured by 2D-SWE could be used to noninvasively identify steatohepatitis and stage fibrosis in NAFLD patients. Moreover, the diagnosis efficiency of the stiffness measured by 2D-SWE could not be influenced by steatosis.
\end{abstract}

Keywords: Nonalcoholic fatty liver disease (NAFLD); nonalcoholic steatohepatitis (NASH); cirrhosis; shear wave elastography (SWE); K-fold-cross-validation

\footnotetext{
^ ORCID: Jie Zhou, 0000-0003-0414-4581; Jinshun Xu, 0000-0003-2420-2320; Qiang Lu, 0000-0002-4057-1997; Xianglan Zhu, 00000002-4918-0626; Binyang Gao, 0000-0002-2974-8691; Huan Zhang, 0000-0002-7292-5489; Rui Yang, 0000-0002-7733-0636; Yan Luo, 0000-0003-2985-1768.
} 
Submitted Jul 06, 2021. Accepted for publication Oct 22, 2021.

doi: 10.21037/qims-21-700

View this article at: https://dx.doi.org/10.21037/qims-21-700

\section{Introduction}

In recent years, the prevalence of non-alcoholic fatty liver disease (NAFLD) has risen $(1,2)$, along with the increasing prevalence of obesity, becoming the most common cause of chronic liver disease worldwide $(3,4)$. The clinical spectrum of NAFLD ranges from simple steatosis (termed nonalcoholic fatty liver, NAFL) to nonalcoholic steatohepatitis (NASH), a progressive form resulting in cirrhosis, hepatic failure, and even hepatocellular carcinoma (HCC) (5-7). Prognosis strongly depends on histological severity (7-9). Therefore, it is important to accurately differentiate NAFL and NASH, which is attributed to formulate the optimum options for monitoring and treatment in NAFLD patients (6).

Although liver biopsy is still considered as the gold standard to evaluate NAFLD, it is an invasive procedure, with rare but potentially life-threatening complications, and is prone to sampling errors $(5,10)$. Several non-invasive methods have been showed good capabilities for diagnosis of NAFL, NASH, and fibrosis, including cytokeratin-18 fragments (CK-18) (11), NAFLD fibrosis score (NFS) (12), fibrosis 4 calculator (FIB-4) (13), and liver stiffness measurement (LSM) based on transient elastography (TE) imaging $(14,15)$. However, none of them can substitute liver biopsy based on the convergent findings from recently critical appraisals (16-18). Furthermore, there has been no consensus on thresholds or strategies of noninvasive methods when trying to avoid biopsy $(5,17,18)$. Consequently, more preferable strategies are urgently needed to improve the capability of the non-invasive measurements for diagnosis of NAFLD subtypes.

Two-dimension real-time shear wave elastography (2D-SWE), is based on the combination of a radiation force induced in tissues by focused ultrasonic beams and a very high frame rate ultrasound imaging sequence capable of catching in real time the transient propagation of resulting shear waves (17). Recently, 2D-SWE is becoming widely used (19-24), and is recommended for the evaluation of liver fibrosis by some guidelines in patients with chronic liver diseases, such as viral hepatitis $(17,25)$. However, except for fibrosis, steatosis would co-exist in the same NAFLD patient.
Thus, the primary aim of this study was to validate the diagnostic accuracy of 2D-SWE for NASH and quality criteria using histopathology as the reference. The second aim of this study was to evaluate and compare the accuracy of 2D-SWE and four previously reported noninvasive serum fibrosis scores (NFS, FIB-4 and BARD scores and APRI) for further staging fibrosis in NASH patients. The influence of steatosis on LSM of 2D-SWE in all NAFLD patients was also been investigated. We present the following article in accordance with the STARD reporting checklist (available at https://dx.doi.org/10.21037/qims-21700).

\section{Methods}

\section{Patients}

The study was conducted in accordance with the Declaration of Helsinki (as revised in 2013). This retrospective study was based on data collected in an electronic database and approved by the Institutional Review Board of West China Hospital of Sichuan University. Informed consent was waived because of the retrospective nature of the study. Patients with biopsy-proven NAFLD were included from September 2015 to February 2021 at West China hospital of Sichuan University. NAFLD was defined as liver steatosis on liver biopsy after excluded the following exclusion criteria: excessive alcohol intake (males were excluded if they consumed more than $30 \mathrm{~g}$ of alcohol per day, and females were excluded if they consumed more than $20 \mathrm{~g}$ per day); co-existing liver diseases: chronic virus hepatitis, autoimmune hepatitis, obstructive cholestasis and acute hepatitis; liver congestion, liver neoplasm, embolus that would affected blood or bile circulation, whatever conditions that might affect liver stiffness; druginduced fatty liver injury and other liver diseases associated with fatty liver, such as hepatolenticular degeneration. In general, the site of liver biopsy puncture usually located in the right liver. To ensure that the region of interest (ROI) of 2D-SWE is close to the site of the liver biopsy puncture, we excluded patients whose puncture site was in the left liver or distant from the V, VII, VIII segment of liver by reading the original images. We also included patients who confirmed 
normal background liver histology by liver mass resection and postoperative histology examination as a normal controlled group, and had negative hepatitis virus markers, no previous history of acute or chronic liver disease, no excessive alcoholic intake, no abnormal signs were found by liver ultrasound examination.

\section{Clinical and laboratory assessments}

Relevant clinical data: sex, age, weight, height, and alcohol intake (g/day), and laboratory results: total bilirubin (TB), blood glucose, triglyceride (TG), alanine transaminase (ALT), aspartate transaminase (AST), alkaline phosphatase (ALP), lactic dehydrogenase (LDH) were collected at the time of liver biopsy. Body mass index (BMI) was calculated as weight $(\mathrm{kg})$ divided by height squared $\left(\mathrm{m}^{2}\right)$.

To evaluate and compare the accuracy of 2D-SWE and four previously reported noninvasive fibrosis scores for further staging fibrosis in NASH patients, the results of 2D-SWE were compared with that of other prediction scores (NFS, FIB-4 BARD, APRI): NFS was calculated according to the following formula: $-1.675+0.037 \times$ age (years) $+0.094 \times \mathrm{BMI}\left(\mathrm{kg} / \mathrm{m}^{2}\right)+$ impaired fasting glycemia or diabetes $($ yes $=1$; no $=0)+0.99 \times$ AST $/$ ALT $-0.013 \times$ platelet $\left(10^{9} / \mathrm{L}\right)-0.66 \times$ albumin $(\mathrm{g} / \mathrm{dL})(12)$. FIB-4 score was calculated by: age $\times$ AST $(\mathrm{U} / \mathrm{L}) /$ platelet count $\left(10^{9} / \mathrm{L}\right)$ $\times \sqrt{ } \operatorname{ALT}(\mathrm{U} / \mathrm{L})(13)$. BARD score was the weighted by the sum of three variables $(\mathrm{BMI}>28=1$ point; AST/ALT ratio $\geq 0.8=2$ points; and diabetes $=1$ point) $(26)$. APRI scoring formulation was AST level/upper limit of normal value $(\mathrm{ULN}) / \mathrm{PLT}\left(10^{9} / \mathrm{L}\right) \times 100(27)$.

\section{US examination and $2 D-S W E$}

All subjects underwent ultrasound examination by SuperSonic Imagine AixPlorer (SuperSonic Imagine, Aix-en-Provence, France) after fast for at least 8 hours. The same proficient sonographer performed ultrasound examinations, 2D-SWE measurement and needle biopsy continuously in one day. The examinees were placed in the supine position with the right-arm maximal extension. Two-dimension ultrasonic examination was first performed before select 2D-SWE mode. The convex probe (SC6-1) was positioned in the right lobe of the liver through the intercostal space (segment V, VIII, or VII) with the transducer at $90^{\circ}$ in relation to the liver capsule in an area free of artifacts and large vessels. The ROI was placed a minimum of 1-2 $\mathrm{cm}$ and a maximum of $6 \mathrm{~cm}$ below the liver capsule. The analysis box was set to at least $10 \mathrm{~mm}$, preferably $15 \mathrm{~mm}$ or more. A round shape is usually chosen. The patients transiently held their breath in a neutral position. Five successful measurements were repeated. Measurements were classified as failed when no or heterogeneous signal was obtained in the ROI. The mean of the five times 2D-SWE measurements expressed in kilopascals $(\mathrm{kPa})$ was used as the representative measurement.

\section{Histopathological assessment}

We performed a biopsy in liver segment V, VII or VIII, close to where the sample box from the 2D-SWE examination was placed through the intercostal plane. Only liver biopsies with a minimum length of $2 \mathrm{~cm}$ and at least six complete portal tracts were considered suitable for further analysis. The liver biopsy specimens were stained with hematoxylin-eosin (HE), Foot and Masson stains. Histopathological diagnosis was conducted by the Department of Pathology of West China Hospital, Sichuan University. Biopsies were reviewed by a junior pathologist and then reviewed by a senior pathologist. Cases with discrepancies were simultaneously reviewed by the original two pathologists and a senior pathologist until a consensus was reached. The diagnostic performance of 2D-SWE was evaluated on based on histopathological inflammation and fibrosis stage according to Kleiner/Brunt et al.'s criteria classification (28-30):

Hepatosteatosis: the presence of hepatosteatosis was defined as steatosis $>5 \%$ of hepatocytes with fatty change. Steatosis was graded $0-3$ based on percent of hepatocytes in the biopsy involved ( 0 is less than $5 \% ; 1$ is $5-33 \% ; 2$ is $33-66 \%$; 3 is more than $66 \%$ ).

Lobular inflammation: grade $0-3$ based on inflammatory foci per $20 \times$ with a $20 \times$ ocular $(0$ is none; 1 is 1 to $2 / 20 \times ; 2$ is up to $4 / 20 \times ; 3$ is $.4 / 20 \times$ ).

Fibrosis: the stage of liver fibrosis was divided into 0-4 stages $(\mathrm{F})$ : $\mathrm{F} 0$ is none; $\mathrm{F} 1$, perisinusoidal/pericellular fibrosis around central veins (zone 3), focally or extensively present; F2, zone 3 perisinusoidal/pericellular fibrosis with focal or extensive periportal fibrosis; F3, zone 3 perisinusoidal/ pericellular fibrosis and portal fibrosis with focal or extensive bridging fibrosis; $\mathrm{F} 4$, cirrhosis.

Pure non-alcoholic fatty liver (NAFL): (I) pure steatosis; (II) steatosis and mild lobular or portal area inflammation without ballooning degeneration (hepatocytes become enlarged and the cytoplasm becomes irregularly clumped 

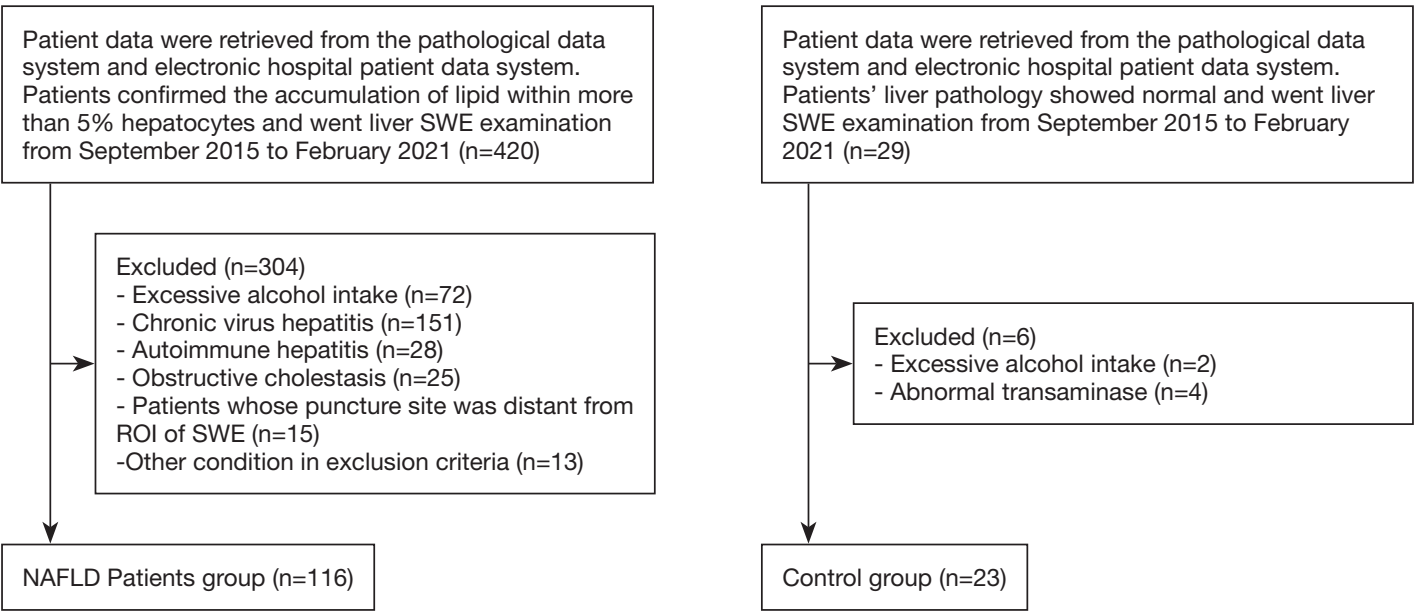

Figure 1 Flow chart of NAFLD patients and normal control group. 2D-SWE, real-time shear wave elastography; NAFLD, nonalcoholic fatty liver disease. ROI, region of interest.

with optically clear, non-vesiculated areas); (III) steatosis and ballooning degeneration without mild lobular and portal area inflammation.

Nonalcoholic steatohepatitis (NASH): early NASH, no or mild (F0-F1) fibrosis (F0 must have ballooning degeneration, mild lobular and portal area inflammation); Fibrotic NASH, significant $(\geq \mathrm{F} 2)$ or advanced $(\geq \mathrm{F} 3)$ fibrosis; NASH-Cirrhosis (F4).

\section{Statistical analysis}

SPSS 20.0 (IBM SPSS Statistics 20.0) statistical software and $\mathrm{R}$ (A language and environment for statistical computing, Vienna, Austria, ISBN 3-900051-07-0, URL http://www.R-project.org) was used for analysis. All continuous variables were confirmed normal distribution or analyzed after normal-scores transformation and were expressed as mean \pm standard deviations (SD), otherwise, median and IQR (25th-75th percentile) were reported. Data were analyzed by using the Student $t$-test, the chisquare test, Fisher's exact test, and analysis of variance, as appropriate. The data got were divided into train sets and test sets, a ratio of $4: 1$ by 5 -fold cross validation of $R$ to calculate the cutoff value, sensitivity (Sen), specificity (Spe), positive predictive value (PPV), negative predictive value (NPV), accuracy (AU) and area under the receiver operating characteristic curves (AUROCs). AUROCs were compared using the DeLong test (31). One-way analysis of variance (One-way ANOVA) with Games-Howell tests was used for multiple comparisons. A P value of 0.05 was considered to be statistically significant.

\section{Results}

\section{Patient characteristics}

The institutional electronic database was collected from September 2015 to February 2021. A total of 420 patients were preliminary selected, and 304 of these patients were excluded for the following reasons: excessive alcohol intake $(\mathrm{n}=72)$, chronic virus hepatitis $(\mathrm{n}=151)$, autoimmune hepatitis $(n=28)$, obstructive cholestasis $(n=25)$, patients whose puncture site was in the left liver or distant from ROI of SWE ( $\mathrm{n}=15)$, and other condition of exclusion criteria $(\mathrm{n}=13)$. In all, 116 NAFLD patients who fulfilled the study criteria were enrolled. We also included 29 patients confirmed normal background liver histology by liver mass resection and postoperative histology examination. Among them, two of the 29 who had excessive alcohol intake (see exclusion criteria), and four of them with elevated transaminase were excluded. Eventually, 23 control group were enrolled. The inclusion and exclusion flow chart of the retrospective study was depicted in Figure 1. The total of 139 patients had an age range from 18 to 77 years, $45.3 \%$ were male. In 116 NAFLD patients group, 21 (18.10\%) patients with no specific inflammation and fibrosis were diagnosed with NAFL, 78 (67.24\%) patients had noncirrhosis NASH, 17 (14.66\%) had cirrhosis NASH. The characteristics and histological findings of the independent study groups are shown in Table 1. Figure 2 showed the 
Table 1 Clinical characteristics and laboratory data of patients

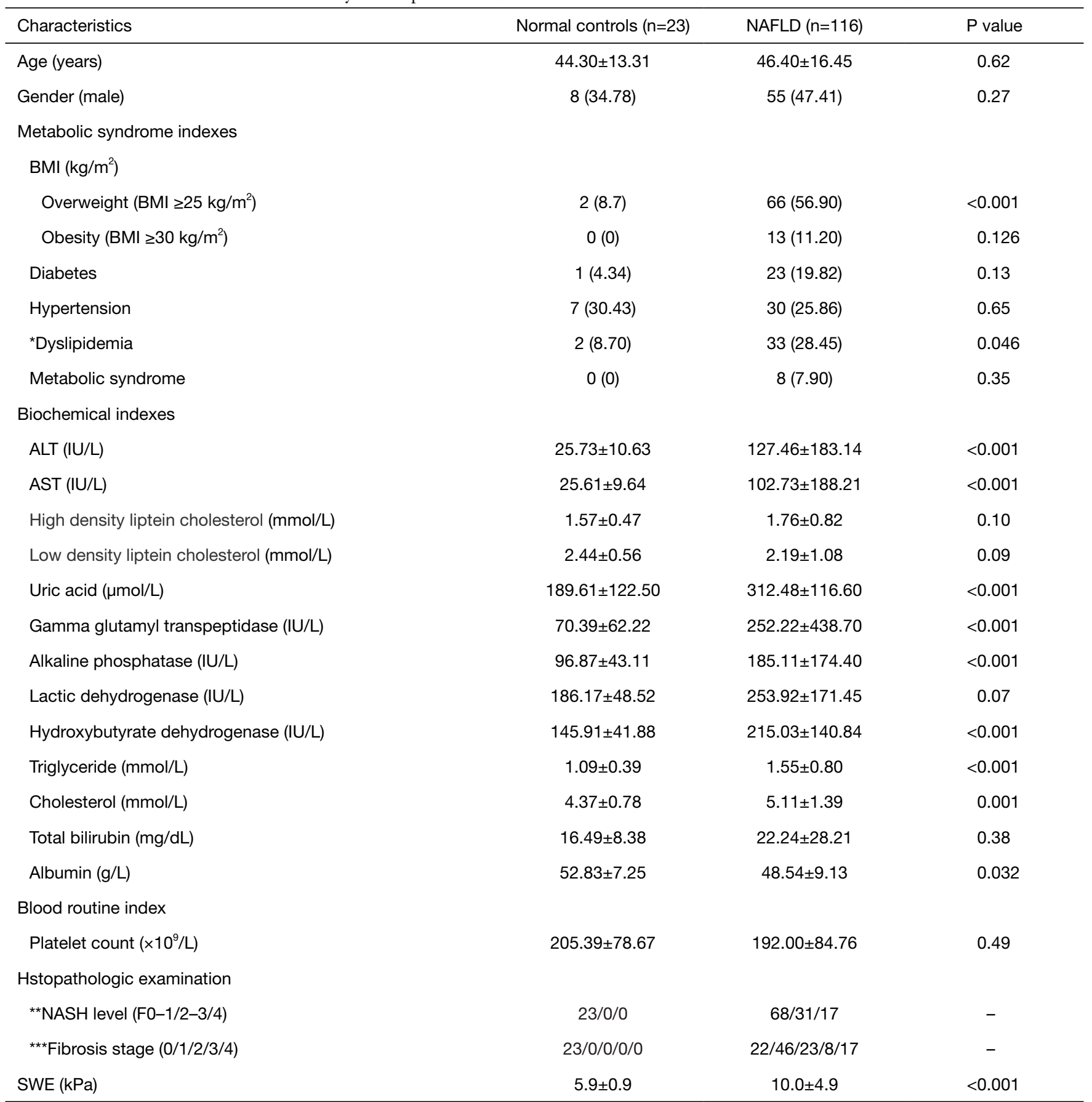

The table shows the mean \pm SD for normally distributed continuous variables or variables that underwent non-normal data distribution transformation, number (\%) for binary variables. *, dyslipidemia: fasting blood triglyceride $\geq 1.7 \mathrm{mmol} / \mathrm{L}$ (150 mg/dL), and/or fasting blood high density liptein cholesterol $<0.9 \mathrm{mmol} / \mathrm{L}(35 \mathrm{mg} / \mathrm{dL})$ (male), $<1.0 \mathrm{mmol} / \mathrm{L}$ (39 mg/dL) (female). ${ }^{\star \star}$, NASH (Nonalcoholic steatohepatitis) level: Early NASH, no or mild (FO-F1) fibrosis (F0 must have ballooning degeneration, mild lobular and portal area inflammation); Fibrotic NASH, significant or advanced fibrosis (F2-F3); NASH-Cirrhosis (F4). ${ }^{* \star *}$, Fibrosis stage: The stage of liver fibrosis was divided into 0-4 stages (F): F0 is none; F1, perisinusoidal/pericellular fibrosis around central veins (zone 3), focally or extensively present; F2, zone 3 perisinusoidal/pericellular fibrosis with focal or extensive periportal fibrosis; F3, zone 3 perisinusoidal/pericellular fibrosis and portal fibrosis with focal or extensive bridging fibrosis; F4, cirrhosis. BMI, body mass index; AST, aspirate aminotransferase; ALT, alanine aminotransferase; $\mathrm{NASH}$, nonalcoholic steatohepatitis; SWE, real-time shear wave elastography. 

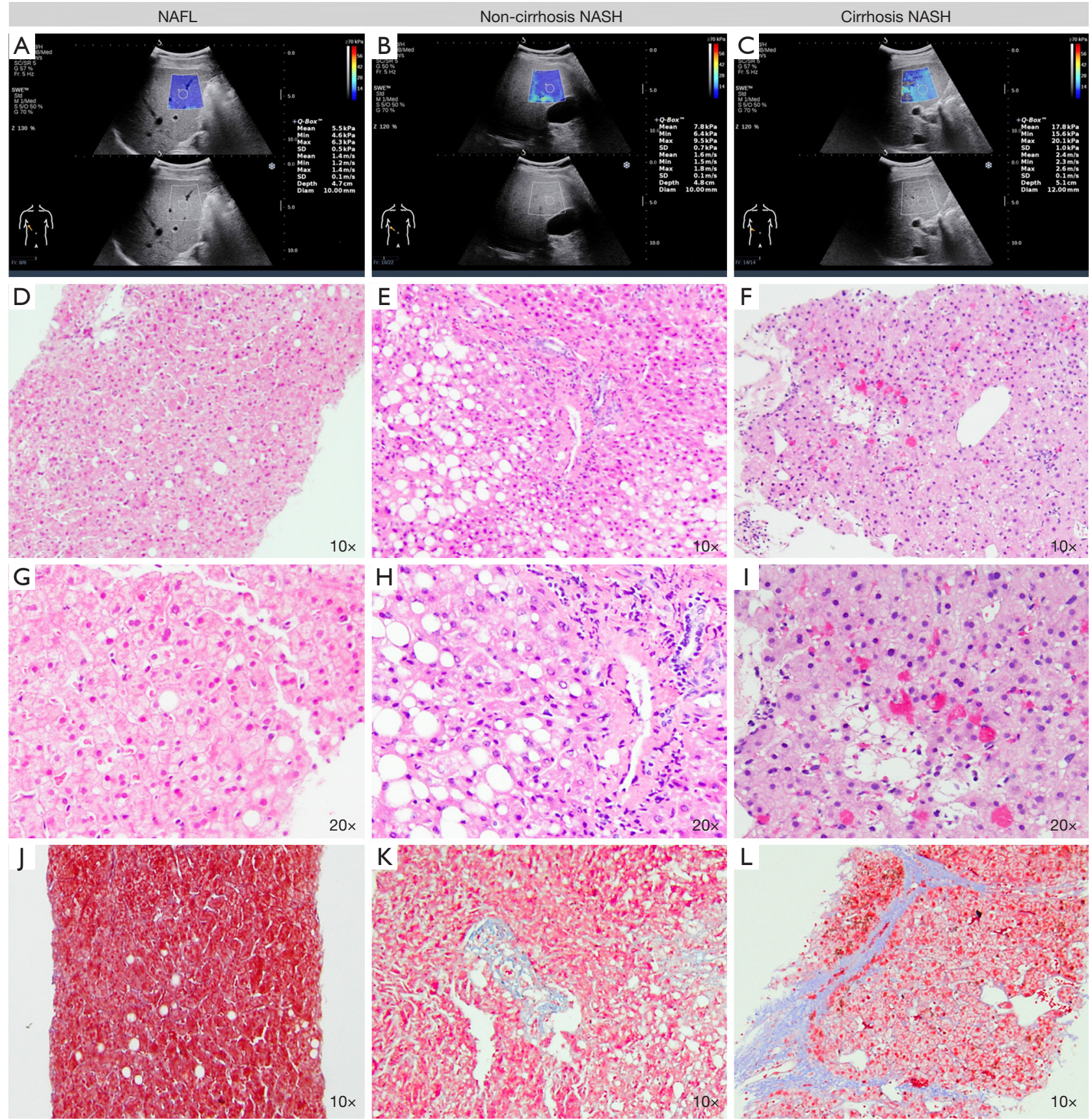

Figure 2 Results of shear wave elastography images (the top row), HE (the second and third rows) and Masson (the bottom row) staining sections in patients with NAFL (the first column), non-cirrhosis NASH (the second column), and cirrhosis NASH (the last column). The mean liver stiffness was 5.5, 7.8, and $13.8 \mathrm{kPa}$, respectively. NAFL, nonalcoholic fatty liver; NASH, nonalcoholic steatohepatitis. 


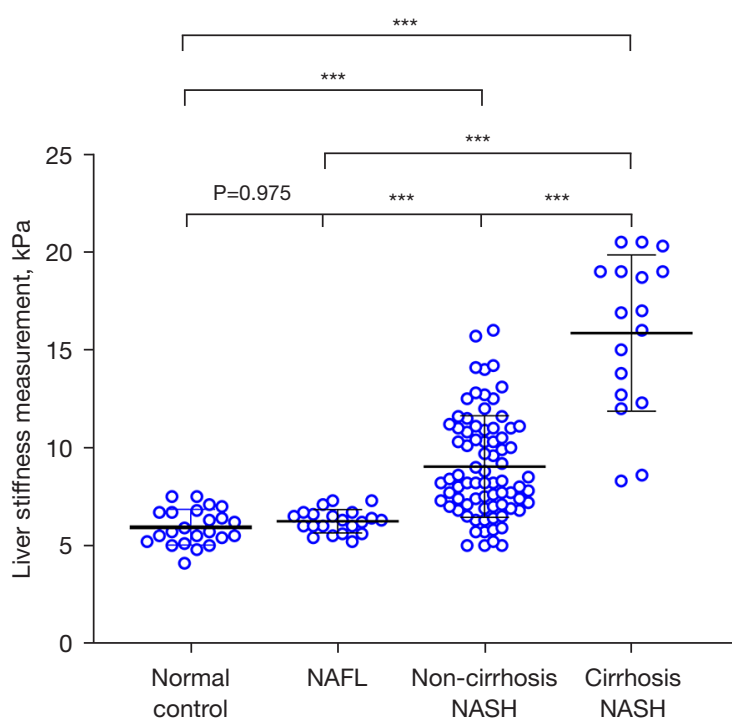

Figure 3 Distribution LSM by 2D-SWE of the normal control, NAFL, non-cirrhosis NASH and cirrhosis NASH group. The LSM $( \pm \mathrm{SD})$ of non-cirrhosis NASH group $(9.0 \pm 2.6 \mathrm{kPa})$ and cirrhosis NASH group $(15.9 \pm 4.0 \mathrm{kPa})$ were higher than both normal control $(5.9 \pm 0.9 \mathrm{kPa})$ and NAFL group $(6.2 \pm 0.6 \mathrm{kPa})$ (all $\mathrm{P}<0.001)$. However, there was no statistical difference between the normal control $(5.9 \pm 0.9 \mathrm{kPa})$ and NAFL group $(6.2 \pm 0.6 \mathrm{kPa})$ $(\mathrm{P}=0.975)$. The bottom of each box, the line in the box, and the top of the box represent the 1st, 2nd, and 3rd quartiles, respectively. The bottom of each box, the line in the box, and the top of the box represent the interquartile range within which $50 \%$ of the values are located. ${ }^{* *}, \mathrm{P}<0.001 .2 \mathrm{D}-\mathrm{SWE}$, two-dimension real-time shear wave elastography; SD, standard deviation; NAFL, nonalcoholic fatty liver; NASH, nonalcoholic steatohepatitis; LSM, Liver stiffness measurement.

example of 2D-SWE images, HE and Masson staining sections.

\section{Predictors of non-cirrbosis NASH and cirrbosis NASH}

The LSM $( \pm \mathrm{SD})$ of normal control, NAFL, non-cirrhosis NASH and cirrhosis NASH group were 5.9 $\pm 0.9,6.2 \pm 0.6$, $9.0 \pm 2.6$ and $15.9 \pm 4.0 \mathrm{kPa}$, respectively, (all $\mathrm{P}<0.001$ ) (Figure 3). Non-cirrhosis NASH group $(9.0 \pm 2.6 \mathrm{kPa})$ and cirrhosis NASH group $(15.9 \pm 4.0 \mathrm{kPa})$ were higher than both normal control $(5.9 \pm 0.9 \mathrm{kPa})$ and NAFL group $(6.2 \pm 0.6 \mathrm{kPa})($ all $\mathrm{P}<0.001)$. However, there was no statistical difference between the normal control $(5.9 \pm 0.9 \mathrm{kPa})$ and NAFL group $(6.2 \pm 0.6 \mathrm{kPa})(\mathrm{P}=0.975)$.
For the diagnosis of non-cirrhosis NASH and cirrhosis NASH by 2D-SWE and calculated optimal cutoff value, the data obtained were randomly divided into train sets and testing sets (ratio of 4:1) by caret package, and then analyzed by pROC package of R. For differentiated non-cirrhosis NASH (5-fold validation results was demonstrated in Figure 4), the mean AUROC $(95 \% \mathrm{CI})$ in train sets was $0.88(0.82$ $0.95)$ when the cutoff was $7.3 \mathrm{kPa}$, whose Sen, Spe, PPV, $\mathrm{NPV}$, and AC were $70.5 \%, 99.4 \%, 66.2 \%, 99.6 \%$ and $80.9 \%$, respectively. In test sets, the Sen, Spe, NPV, PPV, AC were $85.5 \%, 97.8 \%, 82.8 \%, 98.8 \%$ and $89.9 \%$, respectively.

For differentiated cirrhosis NASH by 2D-SWE by k-fold validation (5-fold validation results was demonstrated in Figure 5), the mean AUROC (95\% CI) for 5 -fold cross validation train sets was $0.95(0.89-1.00)$ when the cutoff was $12.6 \mathrm{kPa}$, and its Sen, Spe, PPV, NPV, and AC were $88.1 \%, 91.2 \%, 98.2 \%, 59.2 \%$ and $90.8 \%$, respectively. In test sets, the Sen, Spe, NPV, PPV, AC were $86.7 \%$, 91.0\%, $98.4 \%, 75.6 \%$ and $90.6 \%$, respectively. These results demonstrated 2D-SWE had a significantly high Sen, Spe, $\mathrm{NPV}, \mathrm{PPV}$ and $\mathrm{AC}$ in both train and test sets for diagnosing non-cirrhosis NASH and cirrhosis NASH.

\section{Predictors of fibrosis by $2 \mathrm{D}-\mathrm{SWE}$}

Furthermore, diagnostic performance of 2D-SWE in separating patients' fibrosis stage was evaluated. The mean $\mathrm{LSM}( \pm \mathrm{SD})$ of NAFLD patients from F0 to F4 were $6.1 \pm 0.8$, $8.0 \pm 2.1,10.3 \pm 2.5,11.7 \pm 2.3$ and $15.9 \pm 4.0 \mathrm{kPa}$, respectively. The above data demonstrated a stepwise increase with increasing severity of hepatic fibrosis. Except for F2 and F3 $(\mathrm{P}=0.596)$, the LSM between two adjacent fibrosis stages had statistic difference $(\mathrm{P}<0.05$ for all) (Figure 6).

For diagnosing $\geq \mathrm{F} 2$, the best LSM cutoff was $10.0 \mathrm{kPa}$ in train sets with the AUROC (95\%CI) of $0.86(0.77-0.94)$. For diagnosing $\mathrm{F} 3$ or greater disease, the best LSM cutoff was $11.6 \mathrm{kPa}$ in train sets with the AUROC of $0.89(0.81-0.97)$. The optimal cutoff LSM for diagnosing F4 was $12.6 \mathrm{kPa}$, the AUROC showed 0.90 (0.79-1). The Sen, Spe, PPV, $\mathrm{NPV}, \mathrm{AC}$ and AUROC in train sets were demonstrated in Table 2.

\section{Liver stiffness assessment: comparison of 2D-SWE, NFS, FIB-4, BARD and APRI scores}

Performance of the four noninvasive serum fibrosis scores: NFS, FIB-4, BARD and APRI scores were also assessed by 5 -fold cross validation and compared with $2 \mathrm{D}$-SWE. 

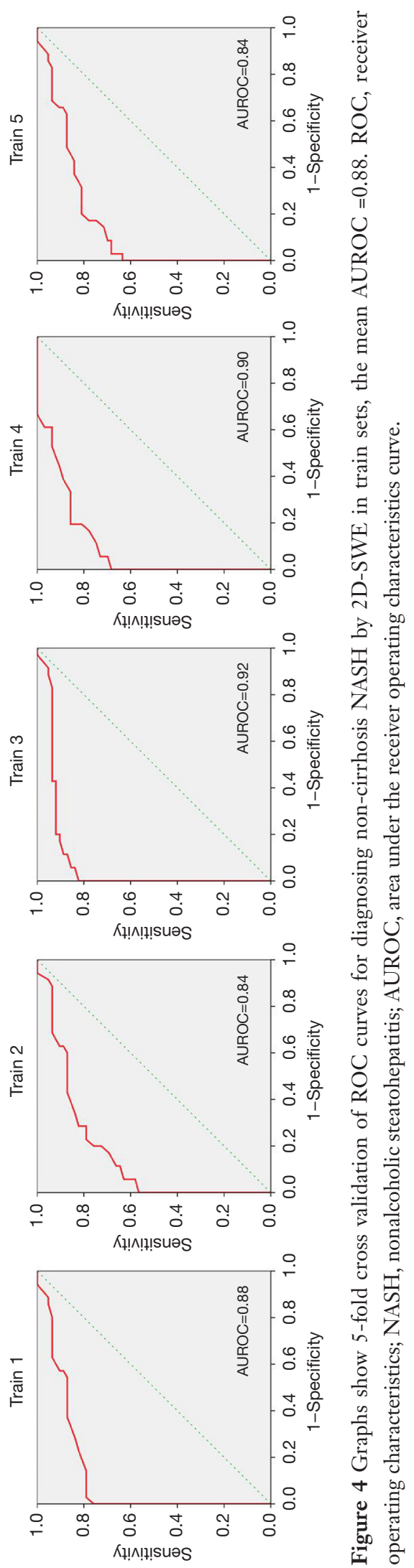
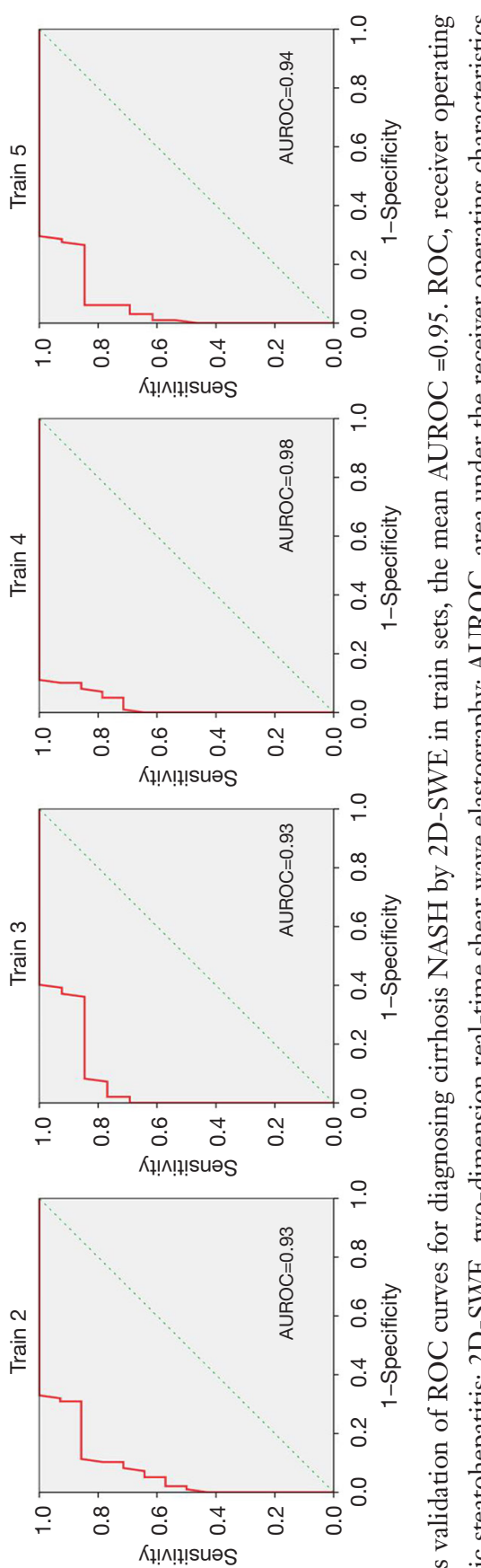

○.

落

范

ฮँ๊

ฐ

离

灵安

节

$\exists$

is

⿵人一

‥

焉常

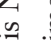

焉

.

蕰.

I

용

है

\%

ชิ

岃: :

.ำ

西

要

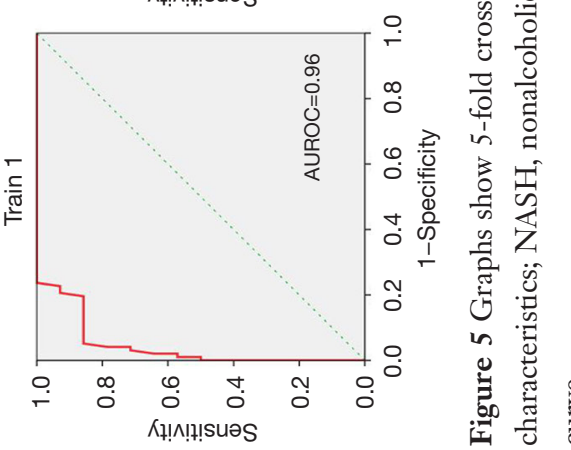


Significantly higher AUROCs were observed for 2D-SWE than for the four noninvasive serum fibrosis scores in the identification of each fibrosis stage $(\geq \mathrm{F} 2: 0.86$ vs. (0.64, $0.61,0.58,0.52) ; \geq \mathrm{F} 30.89$ vs. $(0.70,0.63,0.56,0.51)$; $=\mathrm{F} 4: 0.90$ vs. $(0.66,0.66,0.55,0.51)$; all $\mathrm{P}<0.05)($ Table 3). Nevertheless, the optimal applications for stage fibrosis using NFS, FIB-4, BARD and APRI scores were different:

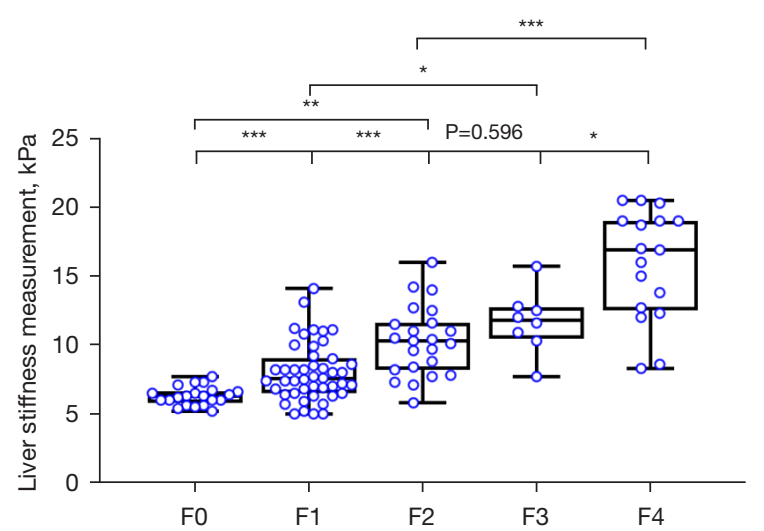

Figure 6 The diagnostic performance of the LSM on 2D-SWE according to fibrosis stage. The mean LSM $( \pm$ SD) of NAFLD patients from $\mathrm{F} 0$ to $\mathrm{F} 4$ were $6.1 \pm 0.8,8.0 \pm 2.1,10.3 \pm 2.5,11.7 \pm 2.3$ and $15.9 \pm 4.0 \mathrm{kPa}$, respectively. Except for F2 and F3, the LSM between two adjacent fibrosis stages had statistic difference $(\mathrm{P}<0.05$ for all). ***, $\mathrm{P}<0.001 ;$ **, $\mathrm{P}<0.01 ;{ }^{*}, \mathrm{P}<0.05$. LSM, Liver stiffness measurement; 2D-SWE, two-dimension real-time shear wave elastography; SD, standard deviation; NAFLD, nonalcoholic fatty liver disease.
APRI is appropriate to $\geq \mathrm{F} 2$, NFS, FIB- 4 and BARD score are more suitable for applying to $\geq \mathrm{F} 3$. In fact, best application of four serum fibrosis scores also showed lower AUROCs for their respective optimum when compared to 2D-SWE. The LSM based on 2D-SWE was significantly superior by Delong test (all $\mathrm{P}<0.05)$ for diagnosis of liver fibrosis stage (F2-4), compared to the NFS, FIB-4, BARD and APRI scores respectively (Figure 7).

\section{The influence of steatosis on LSM}

The nonsteatotic (normal group: $5.9 \pm 0.9 \mathrm{kPa}$ ) and steatotic (NAFL: $6.2 \pm 0.6 \mathrm{kPa}$ ) groups showed no significant difference in LSM $( \pm \mathrm{SD})(\mathrm{P}=0.975)$. The LSM $( \pm \mathrm{SD})$ of NAFL patients with $5-33 \%, 33-66 \%$ and $\geq 66 \%$ of steatosis hepatocytes were $6.3 \pm 0.6,6.2 \pm 0.9$ and $6.4 \pm 1.1 \mathrm{kPa}$, respectively, showing no statistic differences among different steatosis levels $(\mathrm{P}=0.97)$.

\section{Discussion}

The prognosis of NAFLD depends heavily on the histopathological severity (5). Liver biopsy is the traditional gold standard in the differential diagnosis of liver damage, but they cannot be performed as a follow-up test in shortterm intervals, carry a relevant procedure-related risk, and can lead to misclassification due to sampling error $(5,10)$. Therefore, an ideal noninvasive surrogate marker or test is urgently needed.

Several blood biomarkers and score systems have been

Table 2 Accuracy of SWE for diagnosing fibrosis in NAFLD patients

\begin{tabular}{|c|c|c|c|c|c|c|c|c|c|c|c|}
\hline Stage & AUROC $(95 \% \mathrm{Cl})$ & Criteria & Cut-off (kPa) & DA (\%) & Sen $(\%)$ & Spe (\%) & PPV (\%) & NPV (\%) & $-\mathrm{LR}$ & $+\mathrm{LR}$ & DOR \\
\hline \multirow{2}{*}{$\geq \mathrm{F} 2$} & & 95 Sen & 7.3 & 69.2 & 95.8 & 41.3 & 63.0 & 90.5 & 0.05 & 1.63 & 35.45 \\
\hline & & 95 Spe & 11.2 & 76.6 & 58.8 & 95.7 & 93.3 & 68.8 & 0.61 & 13.42 & 22.14 \\
\hline \multirow[t]{2}{*}{$\geq \mathrm{F} 3$} & $0.89(0.81-0.97)$ & $\mathrm{BCV}$ & 11.6 & 86.2 & 80.0 & 88.4 & 71.4 & 92.4 & 0.21 & 6.90 & 31.89 \\
\hline & & 95 Spe & 12.8 & 87.2 & 68.0 & 94.2 & 81.0 & 89.0 & 0.36 & 11.73 & 32.64 \\
\hline \multirow[t]{3}{*}{$=\mathrm{F} 4$} & $0.90(0.79-1.00)$ & $\mathrm{BCV}$ & 12.6 & 89.4 & 76.5 & 92.2 & 68.4 & 94.7 & 0.26 & 9.81 & 38.46 \\
\hline & & 95 Sen & 8.6 & 55.3 & 94.1 & 46.7 & 28.1 & 97.3 & 0.13 & 1.77 & 14.05 \\
\hline & & 95 Spe & 13.5 & 89.4 & 70.6 & 93.5 & 70.6 & 93.5 & 0.61 & 10.87 & 34.56 \\
\hline
\end{tabular}

AUROC, area under the receiver-operating characteristics curve; 95\% Cl, 95\% confidence interval; BCV, the best diagnostic value; DA, diagnostic accuracy; Sen, sensitivity; Spe, specificity; NPV, negative predictive value; PPV, positive predictive value; -LR, negative likelihood ratio; +LR, positive likelihood ratio; DOR, diagnostic odds ratio. 
Table 3 AUROCs of the non-invasive fibrosis tests and SWE

\begin{tabular}{lccc}
\hline \multirow{2}{*}{ Fibrosis test } & \multicolumn{3}{c}{ AUROC (95\% Cl) } \\
\cline { 2 - 4 } & \multicolumn{1}{c}{$\geq$} & $\geq \mathrm{F} 3$ & $\mathrm{~F} 4$ \\
\hline The NAFLD fibrosis score (NFS) & $0.64(0.50-0.76)$ & $0.70(0.55-0.85)$ & $0.66(0.46-0.86)$ \\
FIB-4 & $0.61(0.47-0.71)$ & $0.63(0.49-0.70)$ & $0.66(0.51-0.81)$ \\
BARD score & $0.58(0.45-0.71)$ & $0.56(0.42-0.69)$ & $0.55(0.39-0.70)$ \\
APRI score & $0.52(0.39-0.66)$ & $0.51(0.36-0.66)$ & $0.51(0.33-0.69)$ \\
SWE & $0.86(0.77-0.94)$ & $0.89(0.81-0.97)$ & $0.90(0.79-1.00)$ \\
\hline
\end{tabular}

AUROC, area under the receiver-operating characteristics curve; SWE, shear wave elastography; FIB, fibrosis.

proposed for differential diagnosis of simple steatosis from NASH. Among the clinical and laboratory parameters, the best representative biomarker is cytokeratin- 18 with $66 \%$ of Sen and $82 \%$ of Spe (11). The NAFIC score, calculated from the levels of ferritin, fasting insulin, and type IV collagen $7 \mathrm{~S}$, is useful for the diagnosis of NASH (32). However, the use of laboratory indexes above is uncommon and costly. Moreover, none of them are liver specific and their results may be influenced by changes in clearance and excretion of each individual parameter. Therefore, more longitudinally verifiable data are needed. It is known that the best-studied imaging modality is magnetic resonance elastography (MRE). Chen et al. demonstrated that MRE-based assessments of liver stiffness may have a high diagnostic accuracy (AUC 0.93) for differentiating NASH from simple steatosis, with a cutoff of $2.74 \mathrm{kPa}$ yielding a sensitivity of $94 \%$ with a specificity of $73 \%$ (33). However, the group design utilized in this study lacked the clear histological diagnosis between NASH and not-NASH disease. To date, noninvasive tests have not been validated for diagnosing NASH because of the small number of studies.

2D-SWE is an emerging noninvasive method based on shear waves implemented the diagnostic ultrasound system to provide quantitative analysis of tissue stiffness. In the present study, we evaluated the performance of 2D-SWE in the diagnosis of steatohepatitis and fibrosis in biopsy-proven nonalcoholic fatty liver diseases and compared it with the four fibrosis scores using histopathology as the reference. Liver stiffness measured by 2D-SWE was proved to be a reliable method that can bring potentially steatohepatitis hint and can roughly determine the stage of liver fibrosis. Importantly, liver stiffness measured by 2D-SWE was not influenced by steatosis.

In the present study, we evaluated the performance of
2D-SWE in identifying NASH in NAFLD patients and confirmed the good performance of 2D-SWE to diagnose NASH with an AUROC of $0.88(0.82-0.95)$ at a cutoff of $7.3 \mathrm{kPa}$. To the best of our knowledge, this is the first study to evaluate 2D-SWE in a biopsy-proven NASH, which was deemed to steatosis with lobular and portal area inflammation rather than minor inflammation.

As for staging fibrosis, dozens of noninvasive models composed of blood biochemical biomarkers were reported to be useful, including NFS (12), FIB-4 (13), APRI (26) and enhanced liver fibrosis (ELF) (34). However, APRI and FIB-4 were initially established for patients with hepatitis C virus $(\mathrm{HCV}$ ) infection or HIV/HCV coinfection. ELF need some special tests (such as hyaluronic acid, amino-terminal propeptide of type III procollagen and tissue inhibitor of metalloproteinase-1). In this study, the AUROC of 2D-SWE LSM on detection of fibrosis is superior to those of four scoring systems (NFS, FIB-4, BARD and APRI). This is reasonable because blood markers of the scoring systems are affected by races and diets. Comparing markers of the scoring systems, liver stiffness measured by 2D-SWE has the advantage of imaging liver stiffness in real time while guided by a higher frame-rate B-mode image. Transient elastography (TE) was initially shown to be reliable for assessing fibrosis in patients with chronic hepatitis C (14). TE also was reported to be useful to assess fibrosis in patients with NAFLD $(21,35)$. However, there were some limitations of TE. The first is its one-dimensional imaging that may cannot obtain reliable LSM. Besides, TE has poor performance related to obesity, narrow intercostal space, and ascites (5). 2D-SWE is a more accuracy method of staging fibrosis than TE. Ferraioli et al. showed that 2D-SWE was more accurate than TE in assessing significant fibrosis $(\geq \mathrm{F} 2)$ in chronic hepatitis $\mathrm{C}(21)$. 

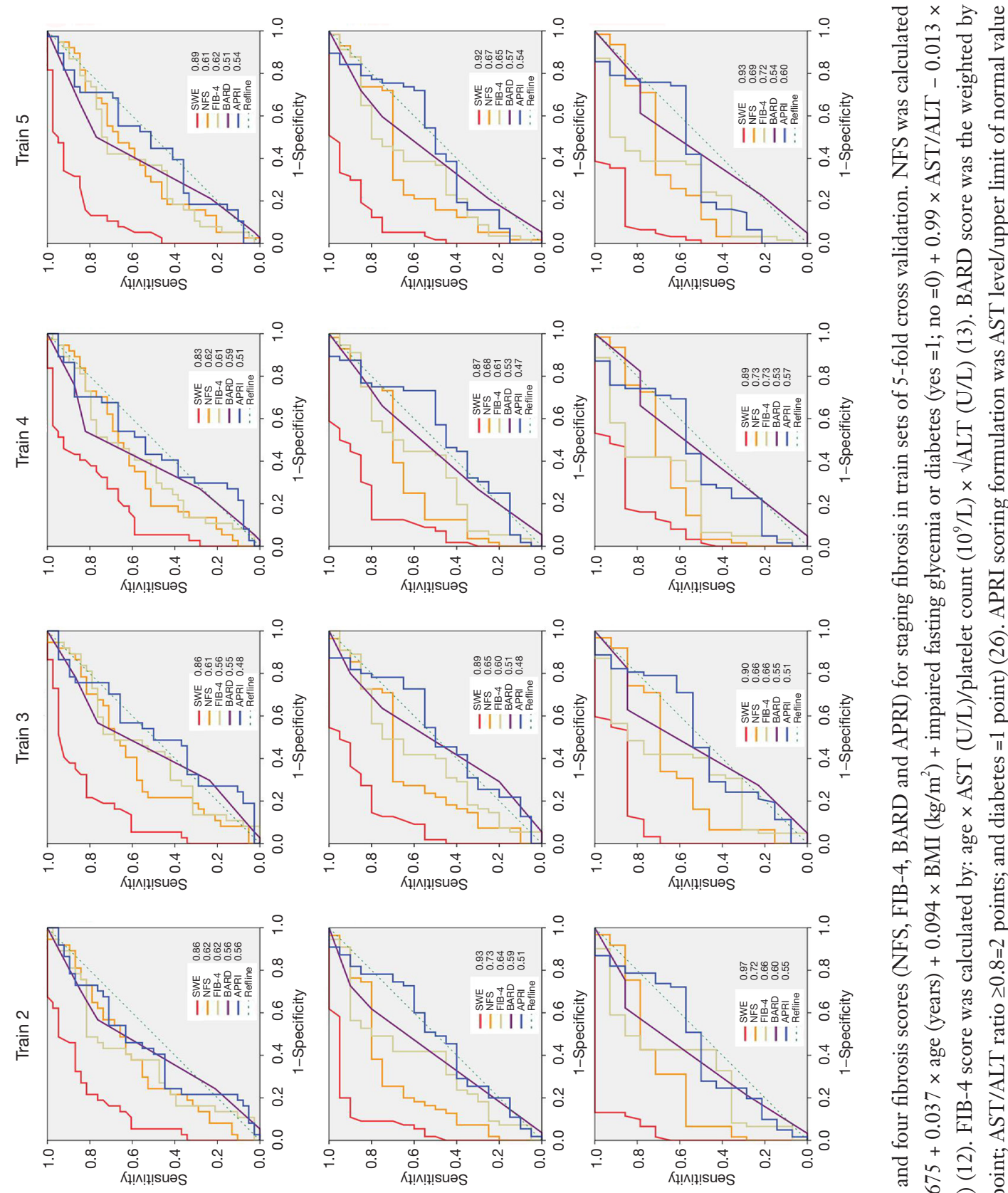

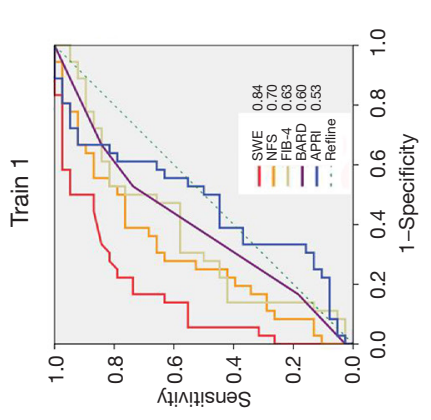

肯

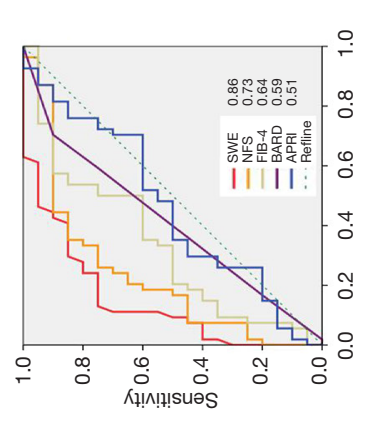

梁

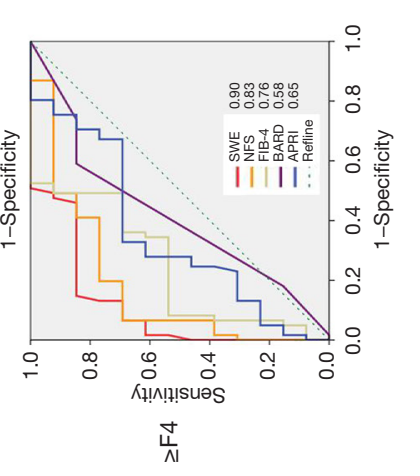

范药

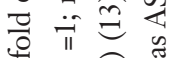

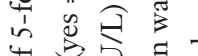
पे

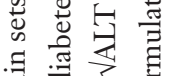
霖 $\times$ $\exists$ Ð

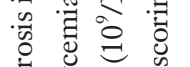

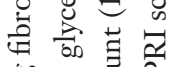

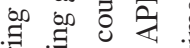
50 . i ๘ 휼 Ð 商言宫 至 + " 元路 일

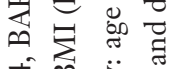
मे $\sum_{1}$ 尚 $\times$ 它苍全 玨志氞 尘完 乙 + क्ष 苟芯芯节 क क 순 节祭产

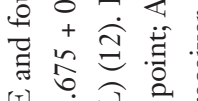

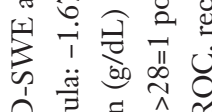

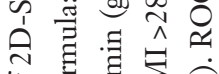
艺

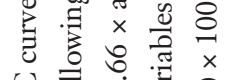

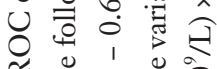
동

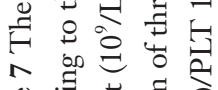
矛艺 寻

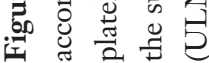


Similarly, a recent meta-analysis studied for NAFLD patients (36) showed the pooled Sen and Spe for diagnosing $\mathrm{F} \geq 2, \mathrm{~F} \geq 3$, and $\mathrm{F}=4$ disease, $\mathrm{TE}$ were $76 \%$ and $65 \%, 75 \%$ and $74 \%, 88 \%$ and $82 \%$, respectively, $2 \mathrm{D}$-SWE were higher than TE, were $85 \%$ and $94 \%, 90 \%$ and $92 \%, 100 \%$ and $86 \%$, respectively.

As for NAFLD diseases, some animal studies have reported that $2 \mathrm{D}-\mathrm{SWE}$ is an efficient technique to differentiate NASH from less severe NAFL $(37,38)$. However, limited human data was available on NAFLD. Samir et al.'s study showed that the use of 2D-SWE provided an AUROC of 0.77 (95\% CI: 0.68, 0.86), with an optimal cutoff of $7.29 \mathrm{kPa}$ (Sen=91.4\%, Spe=52.5\%) for $\geq$ F2 patients with a varied spectrum of liver diseases (NAFLD included) (39). Garcovich et al. assessed 2D-SWE for diagnosing $\mathrm{NASH}$ in pediatric population and reported an AUROC of 0.96 to diagnose $\geq \mathrm{F} 2$ with a cutoff value of $6.7 \mathrm{kPa}(\mathrm{Sen}=87 \%$, Spe=96\%) (40). Ozturk et al. (41) demonstrated the lower threshold of $8.37 \mathrm{kPa}$ than that of $10.0 \mathrm{kPa}$ in this study to diagnose high risk NASH $(\mathrm{F} \geq 2)$. These contrasts in results may be because of the difference in histopathological markers and patients samples. To date, apart from this study, there is no reliably diagnostic threshold to detect NASH in the general population.

In the present retrospective study, no statistical difference was noted in the levels of LSM between the normal control and NAFL groups $(\mathrm{P}=0.975)$. Similarly, Suh et al. used 2D-SWE to study LSM and demonstrated no statistical difference between the normal control group and NAFL group $(\mathrm{P}=0.694)(42)$, suggesting that steatosis would not affect LSM of 2D-SWE. These results were consistent with findings of Samir et al. study that showed steatosis did not correlate with LSM of 2D-SWE (39). However, it cannot be ignored that the influence of severe steatosis on LSM, because fatty attenuation of severe steatosis may lead to 2D-SWE measurement failure (24). Nevertheless, some literatures had inconsistent conclusions. In some transient elastography studies, steatosis has been reported to have an effect on LSM of TE (43-45). Takeuchi et al. found the median liver stiffness values measured using SWE showed a stepwise decrease with increasing hepatic steatosis stage $(\mathrm{P}=0.046)$ (46). Ogawa et al. also investigated the relationship between LSM and histological inflammation score, hepatic fibrosis stage, ballooning score, steatosis analyzed by SWE in rats with NASH, and found that median liver stiffness values measured using SWE showed a stepwise increase with increasing steatosis grade $(\mathrm{P}=0.03)$ (38). However, when discussing the effect of steatosis on LSM, the co-existence of inflammation and fibrosis must be considered, otherwise more confounding factors will greatly affect the results.

One strength of the study is that we consulted clinically concerned NAFLD classifications of EASLEASD-EASO (5): steatosis with minor inflammation is regarded as non-progressive and assigned to the NAFL group, rather than early NASH. So we can make a more obvious diagnosis between NASH and nonNASH. Furthermore, we derived threshold to detect NASH and stage fibrosis. This result can be used for consensus establishment in clinical practice on thresholds or strategies for diagnosing NASH and differentiating fibrosis stage, or further trying to avoid liver biopsy. In addition, we evaluated four fibrosis scoring biochemical systems in Chinese population in this study. However, this study has some limitations. Because of the retrospective nature of the study, we cannot make sure the 2D-SWE measurement and needle biopsy were conducted always by the same ultrasonographer. But we make sure an same ultrasonographer performed 2D-SWE measurement and needle biopsy continuously (at our center ultrasound examinations, 2D-SWE measurement are routinely performed before needle biopsy). This retrospective study was not able to provide information on quality of 2D-SWE measurements and failure rate of 2D-SWE, since data was pooled only for successful $2 \mathrm{D}-\mathrm{SWE}$ readings. Patients in the control group need to meet both the indications of liver biopsy and the conditions of normal liver background, so the number is very limited. For heterogeneous fat deposition, perhaps MRE is more advantageous because SWE LSMs can only represent stiffness of ROI (47). Larger studies are needed to define the effect of these and other confounders and to establish 2D-SWE thresholds for various fibrosis stages in distinct diffuse liver disease.

\section{Acknowledgments}

Funding: The 1.3.5 Project for Disciplines of Excellence, West China Hospital, Sichuan University (ZYJC18008), National Science Foundation of China (NSFC) (81671702, 81701797).

\section{Footnote}

Reporting Checklist: The authors have completed the STARD reporting checklist. Available at https://dx.doi. 
org/10.21037/qims-21-700

Conflicts of Interest: All authors have completed the ICMJE uniform disclosure form (available at https://dx.doi. org/10.21037/qims-21-700). The authors have no conflicts of interest to declare.

Ethical Statement: The authors are accountable for all aspects of the work in ensuring that questions related to the accuracy or integrity of any part of the work are appropriately investigated and resolved. The study was conducted in accordance with the Declaration of Helsinki (as revised in 2013). The study was approved by the Ethics Committee of West China Hospital of Sichuan University (No.2020638), which waived the need for informed consent because all the data used in this study were retrospectively analyzed.

Open Access Statement: This is an Open Access article distributed in accordance with the Creative Commons Attribution-NonCommercial-NoDerivs 4.0 International License (CC BY-NC-ND 4.0), which permits the noncommercial replication and distribution of the article with the strict proviso that no changes or edits are made and the original work is properly cited (including links to both the formal publication through the relevant DOI and the license). See: https://creativecommons.org/licenses/by-nc-nd/4.0/.

\section{References}

1. Loomba R, Sanyal AJ. The global NAFLD epidemic. Nat Rev Gastroenterol Hepatol 2013;10:686-90.

2. Liou I, Kowdley KV. Natural history of nonalcoholic steatohepatitis. J Clin Gastroenterol 2006;40 Suppl 1:S11-6.

3. Masuoka HC, Chalasani N. Nonalcoholic fatty liver disease: an emerging threat to obese and diabetic individuals. Ann N Y Acad Sci 2013;1281:106-22.

4. Pais R, Ratziu V. Epidemiology and natural history of nonalcoholic fatty liver disease. Rev Prat 2012;62:1416-8, 1420-1.

5. European Association for the Study of Obesity (EASO). EASL-EASD-EASO Clinical Practice Guidelines for the management of non-alcoholic fatty liver disease. J Hepatol 2016;64:1388-402.

6. Angulo P. Nonalcoholic fatty liver disease. $\mathrm{N}$ Engl J Med 2002;346:1221-31.

7. Gholam PM, Flancbaum L, Machan JT, Charney DA, Kotler DP. Nonalcoholic fatty liver disease in severely obese subjects. Am J Gastroenterol 2007;102:399-408.
8. Teli MR, James OF, Burt AD, Bennett MK, Day CP. The natural history of nonalcoholic fatty liver: a follow-up study. Hepatology 1995;22:1714-9.

9. Dam-Larsen S, Franzmann M, Andersen IB, Christoffersen P, Jensen LB, Sørensen TI, Becker U, Bendtsen F. Long term prognosis of fatty liver: risk of chronic liver disease and death. Gut 2004;53:750-5.

10. Sanyal AJ, Chalasani N, Kowdley KV, McCullough A, Diehl AM, Bass NM, Neuschwander-Tetri BA, Lavine JE, Tonascia J, Unalp A, Van Natta M, Clark J, Brunt EM, Kleiner DE, Hoofnagle JH, Robuck PR; NASH CRN. Pioglitazone, vitamin E, or placebo for nonalcoholic steatohepatitis. N Engl J Med 2010;362:1675-85.

11. Cusi K, Chang Z, Harrison S, Lomonaco R, Bril F, Orsak B, Ortiz-Lopez C, Hecht J, Feldstein AE, Webb A, Louden C, Goros M, Tio F. Limited value of plasma cytokeratin-18 as a biomarker for NASH and fibrosis in patients with non-alcoholic fatty liver disease. J Hepatol 2014;60:167-74.

12. Angulo P, Hui JM, Marchesini G, Bugianesi E, George J, Farrell GC, Enders F, Saksena S, Burt AD, Bida JP, Lindor K, Sanderson SO, Lenzi M, Adams LA, Kench J, Therneau TM, Day CP. The NAFLD fibrosis score: a noninvasive system that identifies liver fibrosis in patients with NAFLD. Hepatology 2007;45:846-54.

13. Vallet-Pichard A, Mallet V, Nalpas B, Verkarre V, Nalpas A, Dhalluin-Venier V, Fontaine H, Pol S. FIB-4: an inexpensive and accurate marker of fibrosis in $\mathrm{HCV}$ infection. comparison with liver biopsy and fibrotest. Hepatology 2007;46:32-6.

14. Wong VW, Vergniol J, Wong GL, Foucher J, Chan HL, Le Bail B, Choi PC, Kowo M, Chan AW, Merrouche W, Sung JJ, de Lédinghen V. Diagnosis of fibrosis and cirrhosis using liver stiffness measurement in nonalcoholic fatty liver disease. Hepatology 2010;51:454-62.

15. Cassinotto C, Boursier J, de Lédinghen V, Lebigot J, Lapuyade B, Cales P, Hiriart JB, Michalak S, Bail BL, Cartier V, Mouries A, Oberti F, Fouchard-Hubert I, Vergniol J, Aubé C. Liver stiffness in nonalcoholic fatty liver disease: A comparison of supersonic shear imaging, FibroScan, and ARFI with liver biopsy. Hepatology 2016;63:1817-27.

16. Machado MV, Cortez-Pinto H. Non-invasive diagnosis of non-alcoholic fatty liver disease. A critical appraisal. J Hepatol 2013;58:1007-19.

17. European Association for Study of Liver, Asociación Latinoamericana para el Estudio del Hígado. EASLALEH Clinical Practice Guidelines: Non-invasive tests 
for evaluation of liver disease severity and prognosis. J Hepatol 2015;63:237-64.

18. Yang KC, Liao YY, Tsui PH, Yeh CK. Ultrasound imaging in nonalcoholic liver disease: current applications and future developments. Quant Imaging Med Surg 2019;9:546-51.

19. Herrmann E, de Lédinghen V, Cassinotto C, Chu WC, Leung VY, Ferraioli G, et al. Assessment of biopsy-proven liver fibrosis by two-dimensional shear wave elastography: An individual patient data-based meta-analysis. Hepatology 2018;67:260-72.

20. Bavu E, Gennisson JL, Couade M, Bercoff J, Mallet V, Fink M, Badel A, Vallet-Pichard A, Nalpas B, Tanter M, Pol S. Noninvasive in vivo liver fibrosis evaluation using supersonic shear imaging: a clinical study on 113 hepatitis C virus patients. Ultrasound Med Biol 2011;37:1361-73.

21. Ferraioli G, Tinelli C, Dal Bello B, Zicchetti M, Filice G, Filice C; Liver Fibrosis Study Group. Accuracy of real-time shear wave elastography for assessing liver fibrosis in chronic hepatitis C: a pilot study. Hepatology 2012;56:2125-33.

22. Leung VY, Shen J, Wong VW, Abrigo J, Wong GL, Chim AM, Chu SH, Chan AW, Choi PC, Ahuja AT, Chan HL, Chu WC. Quantitative elastography of liver fibrosis and spleen stiffness in chronic hepatitis B carriers: comparison of shear-wave elastography and transient elastography with liver biopsy correlation. Radiology 2013;269:910-8.

23. Zeng J, Liu GJ, Huang ZP, Zheng J, Wu T, Zheng RQ, Lu MD. Diagnostic accuracy of two-dimensional shear wave elastography for the non-invasive staging of hepatic fibrosis in chronic hepatitis B: a cohort study with internal validation. Eur Radiol 2014;24:2572-81.

24. Ochi H, Hirooka M, Koizumi Y, Miyake T, Tokumoto Y, Soga Y, Tada F, Abe M, Hiasa Y, Onji M. Real-time tissue elastography for evaluation of hepatic fibrosis and portal hypertension in nonalcoholic fatty liver diseases. Hepatology 2012;56:1271-8.

25. Ferraioli G, Filice C, Castera L, Choi BI, Sporea I, Wilson $\mathrm{SR}$, et al. WFUMB guidelines and recommendations for clinical use of ultrasound elastography: Part 3: liver. Ultrasound Med Biol 2015;41:1161-79.

26. Harrison SA, Oliver D, Arnold HL, Gogia S, Neuschwander-Tetri BA. Development and validation of a simple NAFLD clinical scoring system for identifying patients without advanced disease. Gut 2008;57:1441-7.

27. Wai CT, Greenson JK, Fontana RJ, Kalbfleisch JD, Marrero JA, Conjeevaram HS, Lok AS. A simple noninvasive index can predict both significant fibrosis and cirrhosis in patients with chronic hepatitis C. Hepatology 2003;38:518-26.

28. Kleiner DE, Brunt EM. Nonalcoholic fatty liver disease: pathologic patterns and biopsy evaluation in clinical research. Semin Liver Dis 2012;32:3-13.

29. Kleiner DE, Brunt EM, Van Natta M, Behling C, Contos MJ, Cummings OW, Ferrell LD, Liu YC, Torbenson MS, Unalp-Arida A, Yeh M, McCullough AJ, Sanyal AJ; Nonalcoholic Steatohepatitis Clinical Research Network. Design and validation of a histological scoring system for nonalcoholic fatty liver disease. Hepatology 2005;41:1313-21.

30. Brunt EM, Janney CG, Di Bisceglie AM, NeuschwanderTetri BA, Bacon BR. Nonalcoholic steatohepatitis: a proposal for grading and staging the histological lesions. Am J Gastroenterol 1999;94:2467-74.

31. DeLong ER, DeLong DM, Clarke-Pearson DL. Comparing the areas under two or more correlated receiver operating characteristic curves: a nonparametric approach. Biometrics 1988;44:837-45.

32. Sumida Y, Yoneda M, Hyogo H, Yamaguchi K, Ono M, Fujii H, Eguchi Y, Suzuki Y, Imai S, Kanemasa K, Fujita K, Chayama K, Yasui K, Saibara T, Kawada N, Fujimoto K, Kohgo Y, Okanoue T; Japan Study Group of Nonalcoholic Fatty Liver Disease (JSG-NAFLD). A simple clinical scoring system using ferritin, fasting insulin, and type IV collagen $7 \mathrm{~S}$ for predicting steatohepatitis in nonalcoholic fatty liver disease. J Gastroenterol 2011;46:257-68.

33. Chen J, Talwalkar JA, Yin M, Glaser KJ, Sanderson SO, Ehman RL. Early detection of nonalcoholic steatohepatitis in patients with nonalcoholic fatty liver disease by using MR elastography. Radiology 2011;259:749-56.

34. Miele L, De Michele T, Marrone G, Antonietta Isgrò M, Basile U, Cefalo C, Biolato M, Maria Vecchio F, Lodovico Rapaccini G, Gasbarrini A, Zuppi C, Grieco A. Enhanced liver fibrosis test as a reliable tool for assessing fibrosis in nonalcoholic fatty liver disease in a clinical setting. Int J Biol Markers 2017;32:e397-402.

35. Guha IN, Parkes J, Roderick P, Chattopadhyay D, Cross R, Harris S, Kaye P, Burt AD, Ryder SD, Aithal GP, Day CP, Rosenberg WM. Noninvasive markers of fibrosis in nonalcoholic fatty liver disease: Validating the European Liver Fibrosis Panel and exploring simple markers. Hepatology 2008;47:455-60.

36. Xiao G, Zhu S, Xiao X, Yan L, Yang J, Wu G. Comparison of laboratory tests, ultrasound, or magnetic resonance elastography to detect fibrosis in patients with nonalcoholic fatty liver disease: A meta-analysis. 
Hepatology 2017;66:1486-501.

37. Kang BK, Lee SS, Cheong H, Hong SM, Jang K, Lee MG. Shear Wave Elastography for Assessment of Steatohepatitis and Hepatic Fibrosis in Rat Models of Non-Alcoholic Fatty Liver Disease. Ultrasound Med Biol 2015;41:3205-15.

38. Ogawa S, Moriyasu F, Yoshida K, Oshiro H, Kojima M, Sano T, Furuichi Y, Kobayashi Y, Nakamura I, Sugimoto K. Relationship between liver tissue stiffness and histopathological findings analyzed by shear wave elastography and compression testing in rats with non-alcoholic steatohepatitis. J Med Ultrason (2001) 2016;43:355-60.

39. Samir AE, Dhyani M, Vij A, Bhan AK, Halpern EF, Méndez-Navarro J, Corey KE, Chung RT. Shear-wave elastography for the estimation of liver fibrosis in chronic liver disease: determining accuracy and ideal site for measurement. Radiology 2015;274:888-96.

40. Garcovich M, Veraldi S, Di Stasio E, Zocco MA, Monti L, Tomà P, Pompili M, Gasbarrini A, Nobili V. Liver Stiffness in Pediatric Patients with Fatty Liver Disease: Diagnostic Accuracy and Reproducibility of Shear-Wave Elastography. Radiology 2017;283:820-7.

41. Ozturk A, Mohammadi R, Pierce TT, Kamarthi S, Dhyani M, Grajo JR, Corey KE, Chung RT, Bhan AK, Chhatwal J, Samir AE. Diagnostic Accuracy of Shear Wave Elastography as a Non-invasive Biomarker of HighRisk Non-alcoholic Steatohepatitis in Patients with Nonalcoholic Fatty Liver Disease. Ultrasound Med Biol 2020;46:972-80.

42. Suh CH, Kim SY, Kim KW, Lim YS, Lee SJ, Lee MG, Lee J, Lee SG, Yu E. Determination of normal hepatic elasticity by using real-time shear-wave elastography.

Cite this article as: Zhou J, Yan F, Xu J, Lu Q, Zhu X, Gao B, Zhang H, Yang R, Luo Y. Diagnosis of steatohepatitis and fibrosis in biopsy-proven nonalcoholic fatty liver diseases: including two-dimension real-time shear wave elastography and noninvasive fibrotic biomarker scores. Quant Imaging Med Surg 2022;12(3):1800-1814. doi: 10.21037/qims-21-700
Radiology 2014;271:895-900.

43. Sánchez-Conde M, Montes Ramírez ML, Bellón Cano JM, Caminoa A, Alvarez Rodríguez F, González Garcia J, Miralles Martín P, Bernardino de la Serna I, Bernardo de Quirós JC, Arribas López JR, Cosín Ochaíta J, Pascual Pareja JF, Alvarez E, Berenguer JB. Impact of liver steatosis on the correlation between liver stiffness and fibrosis measured by transient elastography in patients coinfected with human immunodeficiency virus and hepatitis $\mathrm{C}$ virus. J Viral Hepat 2011;18:e278-83.

44. Kim SU, Kim DY, Ahn SH, Kim HM, Lee JM, Chon CY, Park YN, Han KH, Park JY. The impact of steatosis on liver stiffness measurement in patients with chronic hepatitis B. Hepatogastroenterology 2010;57:832-8.

45. Coco B, Oliveri F, Maina AM, Ciccorossi P, Sacco R, Colombatto P, Bonino F, Brunetto MR. Transient elastography: a new surrogate marker of liver fibrosis influenced by major changes of transaminases. J Viral Hepat 2007;14:360-9.

46. Takeuchi H, Sugimoto K, Oshiro H, Iwatsuka K, Kono S, Yoshimasu Y, Kasai Y, Furuichi Y, Sakamaki K, Itoi T. Liver fibrosis: noninvasive assessment using supersonic shear imaging and FIB4 index in patients with nonalcoholic fatty liver disease. J Med Ultrason (2001) 2018;45:243-9.

47. Zhang QH, Zhao Y, Tian SF, Xie LH, Chen LH, Chen AL, Wang N, Song QW, Zhang HN, Xie LZ, Shen ZW, Liu AL. Hepatic fat quantification of magnetic resonance imaging whole-liver segmentation for assessing the severity of nonalcoholic fatty liver disease: comparison with a region of interest sampling method. Quant Imaging Med Surg 2021;11:2933-42. 